\title{
Cooperativity and control in highly excited Rydberg ensembles - achievements of the European Marie Curie ITN COHERENCE
}

\author{
Matthias Weidemüller ${ }^{\mathrm{a}}$ \\ Physikalisches Institut, Universität Heidelberg Im Neuenheimer Feld 226, \\ 69120 Heidelberg, Germany
}

Received 15 November 2016

Published online 19 December 2016

The European Marie-Curie Initial Training Network "Cooperativity in Highly Excited Rydberg Ensembles - Control and Entanglement (COHERENCE)", funded from 2012 to 2015 by the European Commission, was devoted to promote young researchers in an outermost active field of modern research. The central research topic of the network was the investigation of interactions among electronically highly excited (Rydberg) atoms under extremely controlled conditions, in particular using state-of-the-art techniques of quantum engineering and laser cooling. The field of Rydberg gases, with applications in fundamental science and technology, is at the crossroads between various scientific areas, including atomic physics, condensed matter physics, molecular physics, quantum optics and quantum simulation, surface science, plasma physics, and laser technology. Altogether, 18 European and US universities and research institutions were involved in the network performing research at the highest international level and providing scientific training to the young researchers, among which 20 were directly funded by the European network. As perspective applications of Rydberg systems are already in sight, two optics and laser development companies have joined the network, delivering technological expertise and in-depth insights in business matters. In the four years of the project, the network has significantly shaped the international community in this rapidly growing field of research. More than 100 articles in high-impact international journals were published by the young researchers hired by COHERENCE. As another indicator of its success, COHERENCE has inspired the currently active European Future Emergent Technologies network on "Rydberg Quantum Simulators", funded within the framework of the Horizon 2020 program.

Due to the outstanding, partially spectacular scientific outcomes of the network and the breadth of the topics covered by COHERENCE, we considered it appropriate to summarize the major scientific results of the network in a series of review-style articles, which form the body of this issue of the European Physical Journal Special Topics. The reviews cover essential results of the Ph.D. theses of the early-stage researchers, most of who have graduated by now. We also decided to use this issue for an experiment by encouraging the young scientists of the network to write the articles

\footnotetext{
${ }^{a}$ e-mail: weidemueller@uni-heidelberg.de
} 
independently from their senior supervisors. In this way, we would strengthen the independence of the young researchers and provide higher visibility to their genuine achievements. Most supervisors even refrained from being coauthors of the review articles, and this was not because they were not convinced by the quality of the articles. On the contrary, the outcome of this experiment is formidable, as indicated by the 14 articles in this issue, all of highest standards, all having passed serious peerreviewing. It was inspiring to witness how the additional freedom given to the young researchers motivated them to add a distinctive personal character to the reviews collected in this issue in order to seek their own independence already at an early stage of their career.

The first part of this EPJST addresses technological developments enabling experimental progress with Rydberg gases which goes beyond our imagination at the time when the proposal COHERENCE had been written. It was through this intriguing progress that the many scientific breakthroughs within COHERENCE could be achieved. In a collaboration between the laser company TOPTICA (Germany) and the Joint Quantum Center at Durham University (UK), Kliese et al. present difference-frequency optical combs as an advanced technological tool for measuring optical frequencies and stabilizing laser sources, in particular for applications in Rydberg physics. Naber et al. from the University of Amsterdam (The Netherlands) describe advanced optical techniques for manipulating Rydberg atoms, which are trapped in lattice-like geometries. Henning Labuhn from Institut d'Optique in Palaiseau (France) demonstrates techniques to prepare and detect Rydberg atoms in two-dimensional lattices of arbitrary shape with high fidelity. Busche, Ball and Huillery from the Joint Quantum Centre at Durham University (UK) describe their newly-built experimental apparatus devoted to the study of quantum nonlinear optics employing ultracold Rydberg atoms. Gavyrusev et al. from Heidelberg University (Germany) develop and explore a method to optically detect and image Rydberg atoms using the effects of the Rydberg interactions on a surrounding gas of atoms ("Interaction Enhanced Imaging").

The second part of this EPJST issue is devoted to few-body phenomena involving two, three or four Rydberg atoms. Saßmannshausen, Deiglmayr and Merkt from ETH Zürich (Switzerland) review their recent experimental and theoretical studies of photoassociation of Rydberg long-range molecules and macrodimers. Gaj from Stuttgart University (Germany) gives an overview over the field of ultralong-range Rydberg molecules with special emphasis on the role of external fields on the structure of these exotic molecules and the transition from dimers to polyatomic molecules. Farao, Pelle and Zuliani from the Laboratoire Aimé Cotton in Orsay (France) focus on electric-field induced Förster resonances involving only few (two to four) Rydberg atoms which give rise to enhanced interactions. Bienias from Stuttgart University (Germany) presents a thorough theoretical study of scattering properties and boundstate structure of strongly interacting Rydberg polaritons, which are formed as quasi particles by light propagating through a Rydberg medium under conditions of Electromagnetically Induced Transparency (EIT).

Various questions concerning the frontier of today's understanding of quantum many-body systems form the body of the last part of this issue. Vermersch from University of Innsbruck and the Academy Institute for Quantum Optics and Quantum Information (Austria) presents an in-depth guide on the calculation of interactions between Rydberg atoms in different states and their influence on many-body phenomena such as the Rydberg blockade. Mattioli from the same institution theoretically explores the phase diagram and non-equilibrium dynamics of Rydberg-dressed atoms in one-dimensional lattices. Abdussalam and Gil from the Max-Planck Institute for the Physics of Complex Systems in Dresden (Germany) theoretically investigates the non-equilibrium dynamics in the optical excitation of Rydberg atoms ordered in 
lattice structures under presence of dissipation and laser frequency noise. Valado et al. from INO-CNR and University of Pisa (Italy) experimentally study the role of correlations emerging from Rydberg interactions in ultracold gases. Kohlhoff from the University of Oxford (UK) explores various aspects of the interaction of gas-phase Rydberg atoms with surfaces.

This issue of EPJST provides a comprehensive overview over the achievements of COHERENCE, which would have been impossible without the financial support through the Marie-Curie Actions within the $7^{\text {th }}$ framework programme of the European Commission, which we gratefully acknowledge. I am very grateful to Dr. Danica Subally-Haupt for taking excellent care of all administrative issues within COHERENCE. My warm thanks go to the editorial team of EPJ-ST, in particular Ms. Sabine Lehr from Springer and Mr. Nicolas Puyaubreau from EDP Sciences, for their persistent support (and for their patience). 